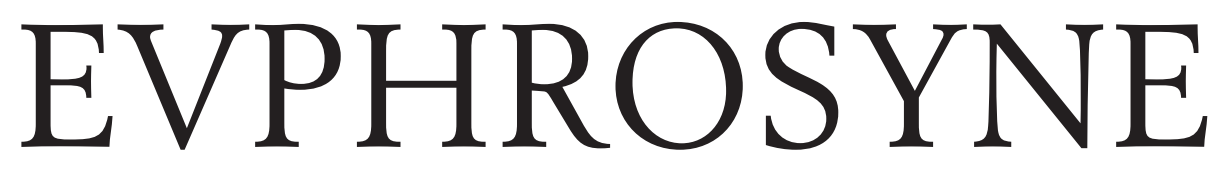
REVISTA DE FILOLOGIA CLÁSSICA

\author{
NOVA SÉRIE \\ VOLUME XLIV
}

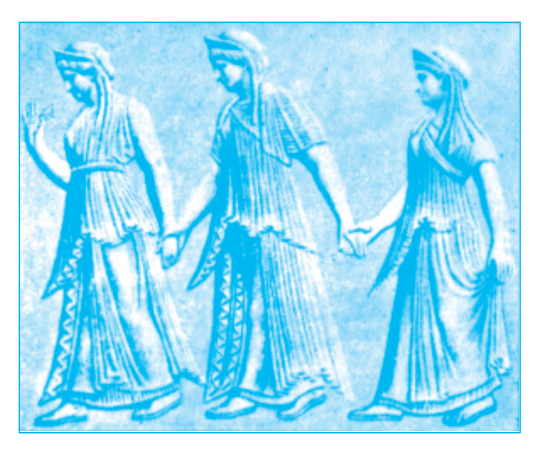

CENTRO DE ESTUDOS CLÁSSICOS

FACULDADE DE LETRAS DE LISBOA 


\section{E V P H R O S Y N E}

REVISTA DE FILOLOGIA CLÁSSICA

$*$

CENTRO DE ESTUDOS CLÁSSICOS

FACULDADE DE LETRAS DE LISBOA

PT - 1600-214 LISBOA

PORTUGAL

e-mail: centro.classicos@letras.ulisboa.pt

sítio electrónico: http://www.tmp.letras.ulisboa.pt/cec

DIRECTORA

Maria Cristina Castro-Maia de Sousa Pimentel

COMISSÃo DE REDACÇÃo

abel do Nascimento Pena, Ana Maria Sanchez Tarrío, Arnaldo Monteiro do Espírito Santo, José Pedro Silva Santos Serra, Manuel José de Sousa Barbosa, Paulo Farmhouse Alberto, Vanda Maria COUTINHO Garrido ANASTÁcio

Conselho Científico

Aires Augusto do Nascimento (U. Lisboa), Carlos Santini (U. Perugia), Carmen Codoñer Merino (U. Salamanca), Emilio SuÁrez de la Torre (U. Pompeu Fabra), JoËl Thomas (U. Perpignan), José Manuel Díaz de Bustamante (U. Santiago de Compostela), Manuel Alexandre Júnior (U. Lisboa), Marc Mayer y Olivé (U. Barcelona), Paolo Fedeli (U. Bari), Thomas Earle (U. Oxford)

\section{Conselho de Arbitragem Científica}

Alberto Bernabé Pajares (U. Complutense de Madrid), Ángel Urbán Fernández (U. Córdoba), Aurelio Pérez Jiménez (U. Málaga), María Adelaida Andres (U. Salamanca), Carlos Morais (U. Aveiro), Carmen Morenilla Talens (U. Valencia), Cristobal Macías Villalobos (U. Málaga), María Elisa Lage Cotos (U. Santiago de Compostela), Eustaquio Sánchez Salor (U. Extremadura), Fabio StoK (U. Roma II - Tor Vergata), Federico Russo (U. Konstanz), Grant A. Nelsestuen (U. Wiscounsin), Graziana Brescia (U. Foggia), Gregorio Rodríguez Herrera (U. Las Palmas de Gran Canaria), Iñigo Ruiz ArZalluz (U. País Vasco), José María Maestre Maestre (U. Cádiz), Jesús-María Nieto Ibáñez (U. León), Joaquim Pinheiro (U. Madeira), Manuel Lopez Muñoz (U. Almería), Nuno Simões Rodrigues (U. Lisboa), RAmiro GonzÁlez Delgado (U. Extremadura), Richard Marshall (U. Oxford), Rosalba Dimundo (U. Bari), Rossana Guglielmetti (U. Milano), Santiago Lopez Moreda (U. Extremadura), Sebastião Tavares de Pinho (U. Coimbra), Silvana Rocca (U. Genova)

Tiragem 500 exemplares

Depósito legal 178089/02

ISSN 0870-0133

\section{PUBLICAÇÃO ANUAL SUJEITA A ARBITRAGEM CIENTÍFICA}

REFERENCIADA EM

L'Année Philologigue | Arts and Humanities Citation Index | Bibliographie internationale de l'Humanisme et de la Remanissance | CSA Linguistics and Language Behavior Abstracts | Dialnet | EBSCO | ERIH Plus | Latindex | Medioevo Latino | SCOPUS 


\section{EVPHROSYNE REVISTA DE FILOLOGIA CLÁSSICA}

NOVA SÉRIE - VOLUME XLIV 



\title{
La apología de la Biblia Regia escrita por Benito Arias Montano: un documento en paradero desconocido
}

\author{
Antonio Dávila PÉRez \\ Universidad de Cádiz \\ antonio.davila@uca.es
}

Como otros muchos repertorios bibliográficos, la Bibliotheca hispana noua de Nicolás Antonio ofrece noticias de documentos de comienzos de la Edad Moderna cuyo paradero, después de cuatro siglos, es completamente desconocido para nosotros. Una de estas noticias informa de una apología que el humanista español Benito Arias Montano (ca. 1525/1527-1598) escribió en español para defenderse de los ataques contra la Biblia Políglota de Amberes, cuya edición había supervisado él mismo. El texto completo dice así:

\begin{abstract}
Apologiae pro se scriptae memoriam tantum reliquit, cuius mentionem reperi nuper in opusculis Pauli Colomesii, ante paucos annos editis Parisiis 1668, in 12: "Ob praeclaram illam - ait - operam in Bibliorum Regiorum editionem insumptam Benedictus Arias uariarum haeresium apud Rom[anum] Pontificem postulatus Apologiam Hispanice scripsit, quam in expugnatione Caletana ante aliquot annos inuentam Angli in patriam suam detulerunt, atque in Oxoniensi bibliotheca, tamquam praetiosissimum $\kappa \varepsilon \iota \eta^{\lambda} \lambda$ เov, etiamnum asseruant. Exoriatur tandem aliquis qui eius editionem procuret!”. Haec ille. ${ }^{1}$
\end{abstract}

Las últimas palabras de este párrafo hacen justicia a la importancia crucial de este texto perdido no solo para la bio-bibliografía de Arias

* Recebido em 22-12-2015; aceite para publicação em 21-03-2016.

** El presente trabajo se ha realizado en el seno del Proyecto de Investigación FFI201564490-P de la DGICYT y de la Red Internacional de Excelencia FFI2015-69200-REDT.

1 N. ANTONIO, Bibliotheca hispana noua siue hispanorum scriptorum qui ab anno MD ad MDCXXXIV floruere notitia, Madrid, Joaquín de Ibarra, 1783, vol. I, p. 210. Nuestra traducción del pasaje es la siguiente: "De una Apología escrita en su defensa solo dejó el recuerdo, de la cual hallé recientemente una mención en los Opúsculos de Paulo Colomesio editados hace pocos años en París en 1668, en $12^{\circ}$ : 'A causa de aquella ilustre obra - dice - emprendida para la edición de la Biblia Regia, Benito Arias fue acusado de varias herejías ante el Pontífice Romano y escribió una apología en español, que los ingleses encontraron hace algunos años en el sitio de Calais y llevaron a su patria, y conservan aún en la Biblioteca de Oxford como un preciosísimo tesoro. Ojalá aparezca alguien que se encargue de la edición de este documento'. Estas son sus palabras". 
Montano, sino también para el estudio de la Filología Bíblica del siglo XVI. A mediados de 1573 se terminó de imprimir en los talleres de Cristóbal Plantino la Biblia Políglota de Amberes, también conocida como Biblia Regia, un monumento a la filología bíblica hebraísta tanto por sus colaboradores como por la metodología empleada. El director de la Políglota comenzó entonces a preparar el terreno para su regreso a casa, precisamente con objeto de protegerse del clima antihebraísta que se respiraba en España tras el encarcelamiento de los profesores salmantinos fray Luis de León, Martín Martínez Cantalapiedra y Gaspar de Grajal, defensores a ultranza del original hebreo de la Biblia. El miedo a un final parecido preocupa Montano, quien comienza dar pasos firmes para defenderse de futuras denuncias, que finalmente se hicieron realidad por obra del profesor de griego de la Universidad de Salamanca León de Castro $^{2}$. Las poderosas influencias del biblista español hacen que se creen dos importantes grupos de apologistas montanianos: uno en los Países Bajos y su entorno, cuyo núcleo duro estuvo conformado por el hebraísta flamenco Francisco Rafelengio, el teólogo lovaniense Juan Harlemio y el orientalista parisino Gilberto Genebrardo ${ }^{3}$; y otro en España, donde son bien conocidas las extensas apologías de Arias Montano y su Biblia firmadas por los humanistas y teólogos Pedro Chacón ${ }^{4}$, Juan del Caño ${ }^{5}$ fray Luis de Estrada ${ }^{6}$.

2 Sobre la polémica recepción del texto de la Biblia Políglota véanse los trabajos de B. Macías Rosendo, La Biblia Políglota de Amberes, Huelva, 1998, págs. XXI-XLII y "El De Arcano Sermone en el marco de la Biblia Políglota de Amberes", en L. Gómez Canseco, F. Navarro Antolín, B. Macías Rosendo (eds.), Libro de José o sobre el lenguaje arcano, Huelva, 2006, pp. 21-42; E. Fernández Tejero, N. Fernández Marcos, "La polémica en torno a la Biblia Regia de Arias Montano", Sefarad, 54, 1994, 259-270; y M. a A. SÁnchez Manzano, Benito Arias Montano. Prefacios de Benito Arias Montano a la Biblia Regia de Felipe II, León, 2006, pp. LII-LXXVII.

${ }_{3}$ Estos autores dedican sus apologías a refutar, principalmente, los ataques del obispo flamenco Guillermo Dámaso Lindano. Véanse en particular las cartas de Genebrardo a Montano con fecha del 25 de noviembre de 1574 en B. MAcías, op. cit., pp. 348-357; de Harlemio a Lindano, escrita hacia junio-julio de 1575, en B. MAcíAs, op. cit, pp. 394-401; y de Francisco Rafelengio a Montano, del 7 de octubre de 1575, en B. MACíAs, op. cit., pp. 444-447.

${ }^{4}$ Pedro Chacón es autor de una larga carta dirigida a León de Castro, publicada por J. F. Domínguez, en su trabajo "Correspondencia de Pedro Chacón (II). Carta n. ${ }^{\circ}$ 3. Pedro Chacón a León de Castro", Ciudad de Dios, 226:1, 2013, 203-244, y comentada por el mismo estudioso en "Correspondencia de Pedro Chacón (III). Comentario a la Carta n. . 3", Ciudad de Dios, 226: 2, 2013, 379-420.

${ }_{5}$ Carta de Juan del Caño a León de Castro, fechada el 7 de enero de [1574] (probablemente, 1575, según J. F. Domínguez, loc. cit., 390), conservada en Ms. A 902, fos 169-172 de la Biblioteca Real de Estocolmo y Ms. Arch. 121, pp. 465-470 del Museo Plantin-Moretus de Amberes. Este documento ha sido publicado por B. MACíAs, op. cit., pp. 314-321. La respuesta de León de Castro a Juan del Caño, con fecha del 24 de enero de 1575, se conserva también en el Museo Plantin-Moretus, Arch. 121, pp. 465-470.

${ }^{6}$ Carta de fray Luis de Estrada a Arias Montano con fecha del 28 de julio de 1576, publicada en B. MACÍAS, op. cit., pp. 456-461; y, seguramente también del mismo año, la "Carta y discurso del maestro Fr. Luis de Estrada sobre la aprobación de la Biblia Regia y sus versiones, y juicio de la que hizo del Nuevo Testamento Benito Arias Montano", publicada en J. Rodríguez DE CASTRo, Biblioteca española. Tomo primero, que contiene la noticia de los escritores rabinos españoles desde la época conocida de su literatura hasta el presente, Madrid, Imprenta Real de la Gaceta, 1781, pp. 649-660 (impresión facsímil en L. Esteban, IV Centenario de fray Luis 
Aun constatando la existencia de todos estos documentos en defensa de la Biblia Políglota escritos por correligionarios de Arias Montano, por extraño que pueda parecer no se ha conservado ninguna apología en español de la Políglota procedente de la pluma del protagonista de la polémica. Por ello Nicolás Antonio se hacía eco de las palabras de Paulo Colomesio y deseaba, con razón, que alguien pusiera a disposición del público erudito la edición de un manuscrito que calificaba, con justicia, como un preciosísimo tesoro.

Para arrojar un poco más de luz sobre el misterioso documento tan encarecido en la nota bibliográfica de Nicolás Antonio, este trabajo abordará un tripe objetivo: (1) conjeturar el contenido y las principales líneas argumentales de la apología montaniana; (2) reconstruir la historia de la transmisión del manuscrito citado por Nicolás Antonio; (3) presentar los datos que hemos podido reunir sobre la búsqueda incesante de este documento realizada por una larga serie de estudiosos desde el siglo XVII hasta nuestros días.

\title{
I. Apologiae pro se scriptae memoriam tantum reliquit
}

Habida cuenta de la importancia de la apología de Arias Montano, sería esperable hallar abundantes referencias a la misma en la correspondencia privada del biblista español. Por desgracia, esto no es así. De hecho, tan solo hemos localizado la que, a nuestro juicio, podría ser una referencia a dicho documento en una de las muchas piezas sueltas del comercio epistolar de la República de las Letras española del siglo XVI. En una carta de Antonio Agustín a un destinatario no citado, con fecha del 21 de octubre, se puede leer el siguiente pasaje:

\begin{abstract}
La apología en defensión de la lengua s[ant]a creo será bien menester en estos t[iem]pos, según que entiendo han sido algunos perseguidos por defendella y por un libro de León de Castro sobre Esaías, veo muchos fundamentos y ocassiones que toman para perseguir los que declaran la sagrada scritura conforme a la letra haebrea y a los rabines y otros intérpretes que la siguen; llamando a los christianos judaizantes por esta causa, pretendiendo que la de los LXX y de los griegos es más christiana y verdadera interpretación, y sobre todas la Vulgata, conforme al canon del Concilio de Trento. Y oigo dezir que sobre este artículo están pressos Martínez y fray León y otros. Y pues este peligro se muestra, V[uestra] M[erced] mire muy bien lo que escrive, y comunique primero con muchos hombres doctos y sabios p[ar]a no herrar, o dexe la empresa p[ar]a otros tiempos. ${ }^{7}$
\end{abstract}

de Estrada. Monasterio de Santa María de Huerta, 1983, pp. 343-360) y posteriormente en R. MuÑ̃z, Carta apologética del sabio cisterciense Fr. Luis de Estrada por el mérito y arreglo de la célebre Biblia Regia de Arias Montano y sus versiones, Burgos, Joseph des Navas, 1794.

7 Cf. J. Carbonell I Manills, Epigrafía i numismática a l'epistolari d'Antonio Agustín (1551-1563), Tesis doctoral, Universidad Autónoma de Barcelona, 1991, pp. 614-615. Este trabajo está digitalizado en http://hdl.handle.net/10803/5551. La minuta de la carta de Agustín se encuentra en la Biblioteca Nacional de España, Ms. 1854, fos $36^{\mathrm{r}}-37^{\mathrm{v}}$ (el manuscrito presenta también una paginación antigua, pp. 63-65). 
Joan Carbonell, primer editor de este documento, proponía como destinatario de la carta a Benito Arias Montano, seguido de un signo de interrogación, suscribiendo así la identificación de destinatario ya propuesta en el inventario de manuscritos de la Biblioteca Nacional ${ }^{8}$ y aportando algunos argumentos propios. La extensa bibliografía montaniana que ha visto la luz en los últimos años avala esa hipótesis, como desarrollaré en otro trabajo. Por ahora lo que nos interesa aquí estudiar es esa referencia a una "apología en defensión de la lengua santa", que Agustín considera necesaria, advirtiendo al mismo tiempo a su autor de los peligros que puede acarrear tal empresa en aquellos tiempos revueltos. La referencia que hace Agustín de este documento demostraría, en primer lugar, que Arias Montano ya tenía el proyecto en mente, si no escrito en parte, en octubre de 1576, coincidiendo en las fechas con esas otras defensas redactadas por sus partidarios; y, en segundo lugar, que Montano estaba sometiendo algunas ideas de su tratado apologético al juicio de otros sabios de su tiempo.

Resulta también obligado plantearse si el escrito referido por Antonio Agustín ("la apología en defensión de la lengua santa") es el mismo que el citado en la nota de Nicolás Antonio ("Apologiae [hispanice] pro se scriptae..."). Nos inclinamos a pensar que esto es así, porque Arias Montano y su equipo de colaboradores siempre basaron su defensa de la Biblia Políglota en la puesta en valor de la lengua hebrea y de los textos originales de la Biblia (hebreo y griego) para la edición y hermenéutica de la Sagrada Escritura.

La prueba más evidente de que la defensa de la lengua hebrea y la defensa de sí mismo como director de la Biblia Políglota eran una misma cosa para Arias Montano es una larga carta latina que el humanista español remitió a finales de 1574 a Gilberto Genebrardo que precisamente titula, en el endoso del borrador, "Pro hebraicis exemplaribus et lingua" ("En defensa de la lengua y de los ejemplares hebreos")9. Los argumentos que esgrime Montano en esta extensa apología latina se repiten de forma recurrente en los otros escritos apologéticos de la Políglota procedentes de las plumas de Rafelengio, Harlemio, Chacón, Luis de Estrada, Juan del Caño y del propio Genebrardo. Por ello creemos que estas mismas ideas, que resumiremos a continuación en tres puntos básicos, debían de formar también parte de los pilares argumentales de la desaparecida apo-logía de la que habla Nicolás Antonio:

1) Seguir atacando los originales hebreos de la Biblia desde la premisa de que han sido corrompidos por los judíos puede devenir en un rechazo del texto sagrado en todas las lenguas, hecho completamente inadmisible, porque supone posicionarse claramente en el bando de los mahometanos, quienes abogaban por abstenerse por completo

8 Donde se conjeturaba, también entre interrogantes, el nombre de Arias Montano. Cf. Inventario general de manuscritos de la Biblioteca Nacional. V (1599-2099), Madrid, 1959, p. 258.

9 Vid. A. Dávila Pérez, "Pro hebraicis exemplaribus et lingua: carta latina inédita de Benito Arias Montano a Gilberto Genebrardo (BNE, MS. 149)”, Ágora. Estudos Clássicos em Debate, $17: 1,2015,337-412$. 
de la lectura de los originales bíblicos hebreos y griegos puesto que presuntamente estaban corrompidos.

2) El valor del estudio de las lenguas originales de la Biblia es indiscutible. El texto sagrado, que en los tiempos de Montano era objeto de ataques, había sido preservado en sus lenguas originales a lo largo de los siglos para transmitir la doctrina de la verdadera piedad. Un sector de la filología bíblica del XVI aducía que el estudio del hebreo no tenía ningún valor; quienes ignoran una materia - alegan los hebraístas - no son competentes para valorar el provecho de la misma.

3) Los estudiosos que defienden que el original hebreo de la Biblia ha sido corrompido por los judíos o no saben hebreo ${ }^{10}$ o saben solo lo necesario para ir cotejando el hebreo con las versiones griegas y latinas y comprobar si varía o falta algo. Además, puesto que estos detractores de la lengua hebrea desconocen todas las versiones latinas de la Biblia existentes desde el pasado, no pueden decir que un texto hebreo difiere $\mathrm{u}$ omite algo con respecto a todas las versiones latinas. Aunque los originales hebreos se aparten en algún pasaje de la Vulgata, proclamada como la versión auténtica del texto sagrado por el Concilio de Trento ${ }^{11}$, ninguno de estos lugares son contrarios $\mathrm{u}$ opuestos al texto latino ${ }^{12}$. Las variantes de significado no pueden llamarse contradicciones: la lengua hebrea es muy rica en matices y no puede ser recogida palabra por palabra ni en latín ni en griego.

Lamentablemente, no hemos hallado noticias sobre más testimonios de la apología de Arias Montano, aparte de la copia manuscrita de la que habla Nicolás Antonio. El manuscrito de la Biblioteca Real de Estocolmo A 902 reúne la colección más importante de documentos para comprender la polémica que rodeó a la Biblia Políglota de Amberes; ahí el lector curioso puede seguir la historia de las acusaciones de León de Castro y Lindano contra la Políglota y el relato de las gestiones de Arias Montano para contrarrestarlas $^{13}$. En este legajo no ha quedado copia ni huella alguna de la apología de Montano, aunque, por ser la recopilación obra del propio biblista español, es de suponer que toda esta documentación epistolar debió de constituirse en fuente primaria e inmediata para la redacción posterior del escrito perdido en defensa de la Políglota de Amberes.

10 Esta acusación de ignorancia del hebreo también se puede leer en la carta de Pedro Chacón a León de Castro (1575 - mayo de 1576), publicada por J. F. DomíngUEz, loc. cit., 237.

11 En la sesión cuarta del 8 de abril de 1546 del Concilio de Trento se promulgó el Decretum de editione et usu sacrorum librorum.

12 Este es uno de los argumentos fuertes en el documento donde Montano trata de conseguir de la Universidad de Lovaina una ampliación del decreto tridentino sobre la Vulgata; cf. A. Dávila Pérez, Benito Arias Montano. Correspondencia conservada en el Museo PlantinMoretus. Alcañiz-Madrid, Instituto de Estudios Humanísticos - Editorial Laberinto - CSIC, 2002, vol. I, pp. 171-173.

13 Esta correspondencia ha sido publicada por B. MACíAs RosENDO en su ya citado trabajo La Biblia Políglota de Amberes en la correspondencia de Benito Arias Montano, Huelva, 1998. 


\section{Fuentes para la historia de la transmisión del manuscrito}

El hilo del que hay que comenzar a tirar para conocer algo más sobre la historia de la transmisión de la copia de la apología montaniana citada en la nota biográfica de la Bibliotheca hispana noua es la relación que sobre la misma escribe Nicolás Antonio. Analicemos, en principio, las fuentes aducidas por el bibliófilo español. Antonio, como se ha dicho, declara haber leído la referencia a la Apologia en unos opúsculos de Paulo Colomesio publicados en París en 1688. Y, en efecto, tras localizar esta obra, hemos podido comprobar que el texto de Colomesio coincide ad pedem litterae con la cita de Nicolás Antonio:

Ob praeclaram illam operam in Bibliorum Regiorum editionem insumptam Benedictus Arias uariarum haeresium apud Rom[anum] Pontificem postulatus Apologiam Hispanice scripsit, quam in expugnatione Caletana ante aliquot annos inuentam Angli in patriam suam detulerunt, atque in Oxoniensi bibliotheca, tamquam praetiosissimum $\kappa \varepsilon \mu \eta \dot{\lambda} \lambda_{10}$, etiamnum asseruant. Exoriatur tandem aliquis qui eius editionem procuret! ${ }^{14}$

Pero el problema es que Colomesio no cita la fuente de esta información, y por ello comenzamos a sospechar que debía de haber una fuente anterior de la que Colomesio tomó la referencia sin citarla, práctica que, como sabemos, era común entre los humanistas. En primer lugar, revisamos el primer catálogo de la Bodleian, la biblioteca de la Universidad de Oxford, publicado en 1605 por Thomas James (1573-1629) ${ }^{15}$; en este primer repertorio no hay ninguna referencia a la apología de Montano.

Pero entre las obras del mismo James se encuentra también un libro titulado A Treatise of the Corruption of Scripture, Councils, and Fathers, by the Prelats, Pastors, and Pillars of the Church of Rome, for Maintenance of Popery. Together with a Sufficient Answer unto James Gretser and Anthony Possevine, Jesuits, and the Unknown Author [May, a Priest] of the Grounds of the Old Religion \& the New. Divided into Five Parts, publicado por vez primera en Londres 1612. Y en dicho tratado, al final de la tercera parte, se puede leer este texto definitivo ${ }^{16}$ :

Arias Montanus, a man, that for his sincere and upright dealing about the Kings Bible, procured unto himselfe much hatred and ill will. Insomuch that hee was constrained to write an Apologie of set purpose, for to prove his integritie (wherein is contained a full satisfaction to all his aduersaries obiections, and the whole historie of his troubles; the beginning, successe and progresse of that costly work) written in the Spanish tongue, never yet printed. The copie was found at the surprise of Cales: and by Gods providence lighting

14 Pauli Colomesii opuscula, Parisiis, apud Sebastianum Mabrecramoisy, 1668, pp. 76-77.

15 Catalogus librorum bibliothecae publicae quam uir ornatissimus Thomas Bodleius eques auratus in Academia Oxoniensi nuper instituit. Cum quadruplici elencho expositorum S. Scripturae, Aristotelis, iuris utriusque et principum medicinae, Oxford, apud I. Barnesium, 1605. Existe edición facsímil (Oxford, Clarendon Press, 1986).

16 Parte 3, pág. 43. 
into the hands of a very bountifull and studious Doctor, as any wee have of his meanes in the Vniversitie (neither to flatter him nor dispraise others) was by him laid up in our rich Cabinet, amongst other Jewels of the like price.

En el margen se añade la información de que el doctor a quien se debe la conservación del manuscrito es "Mr. Dr. Ryves, the warden of the New College”, personaje que hemos identificado con George Ryves (1561-1613), elegido alcalde del New College de Oxford en 1599.

La referencia de James es más completa que las de Colomesio y Antonio, e incluso aporta información sobre el contenido de la apología: satisfacción total a las objeciones de los adversarios (cuyas líneas argumentales básicas hemos expuesto en el primer apartado del presente artículo) y relación detallada de la historia de sus problemas, el principio, éxito y progreso de aquella costosa obra (es decir, la Biblia Políglota). Todo ello parece indicar que, al menos hasta 1612, se podría tener acceso a la referida apología.

Ahora bien, ¿de dónde habían traído los ingleses este documento manuscrito? James escribe que el manuscrito fue "encontrado" (eufemismo) "at the surprise of Cales", topónimo que a mi juicio contiene un error de imprenta. Cales, como tal, resulta extraño como topónimo en inglés de la conocida ciudad francesa del Canal de la Mancha. Si James se estuviera refiriendo a esa ciudad debía haber escrito Calais. Pero ni hubo un asedio inglés de Calais a finales del siglo XVI, fecha en que el documento fue depositado por Ryves en la Bodleian, ni sería muy probable que allí se conservara una copia del documento montaniano. Sin embargo, sí hubo un conocido episodio bélico en el verano de 1596: el saqueo de Cádiz por los ingleses. Tras dos semanas de pillaje, los ingleses regresaron a su patria con un elevado número de rehenes y un inmenso botín que incluía libros y manuscritos. En Cádiz, muy cerca de Sevilla, donde vivía Montano en la etapa final de su vida, podría fácilmente haber una copia de este documento en manos de alguno de sus corresponsales de la Catedral de Cádiz: tenemos noticias, por ejemplo, de Martín de Haya, racionero de la Iglesia de Cádiz, que mantuvo correspondencia erudita con Arias Montano, intercambiando epitafios y monedas romanas, y que fue llevado como rehén por los ingleses en julio de 1596 (algunos rehenes, por cierto, no serían liberados hasta julio de 1603) ${ }^{17}$.

Así pues, la copia de la apología montaniana que llegó a Oxford procedía de Cádiz y no de Calais: en el tratado de James el topónimo Cádiz fue transcrito erróneamente, por alguna de las muchas razones que explican este tipo de faltas, como Cales $^{18}$. En consecuencia, Colomesio, que tomó la refe-

17 J. DE la Concepción, Emporio del orbe, Cádiz ilustrada: investigación de sus antiguas grandezas, Amsterdam, 1690, pp. 119-120 (+121-122).

18 En el curso de nuestra investigación hemos dado con un repertorio titulado The British Librarian. Exhibing a Compendious Review or Abstract of Our Most Scarce, Useful and Valuable Books in All Sciences, as Well in Manuscript as in Print, Londres, 1737, donde se reseña algunos de los más preciosos libros y manuscritos en todas las ciencias. En la página 205 se resume el contenido de la obra de James y se transmite la noticia sobre la apología de Montano, corrigiendo "Calais" por "Cádiz", corrección que avala nuestra hipótesis y que ha pasado inadvertida a toda la investigación posterior. 
rencia de James y la tradujo al latín, optó por el topónimo latino de Calais (Caletana) y Nicolás Antonio, que bebió de Colomesio, transmitió el mismo topónimo a la mayor parte de la bibliografía española posterior.

\title{
III. La búsqueda de la apología de Arias Montano
}

Desde que esta noticia fue publicada por James y recogida, al menos, por Colomesio y Nicolás Antonio, ha habido una larga cadena de estudiosos españoles y del resto de Europa que han tratado de localizar este manuscrito, siglo tras siglo, en las bibliotecas de Oxford.

En la segunda mitad del siglo XVII el teólogo inglés John Mill (1644/51707), quien llegó a ser director del Saint Edmund Hall de Oxford, respondía a una consulta bibliográfica en una carta sin fecha y sin destinatario expreso. La ficha bibliográfica de la Bodleian Library conjetura que la carta iba destinada al secretario o asociado de William Sancroft, arzobispo de Canterbury entre 1678 y 1690, a quien Mill saluda de forma indirecta al final de su misiva ${ }^{19}$. La consulta sobre Montano seguramente procedía del propio Sancroft, pues la carta de Mill se encuentra entre sus papeles. A Mill se le había preguntado si podría localizar la conocida apología de Montano en las bibliotecas de Oxford y su respuesta fue la siguiente ${ }^{20}$ :

\begin{abstract}
As to what you write of Arias Montanus, his Apology, whether ever there was any such book I cannot tell. I am sure there is no MS of his now in the Library. But I persuade myself, having not as yet consulting [sic] the passage where it is cited by Dr James ${ }^{21}$, that you are under some mistake. Had there been any MS then in the Library, James would have put it in his printed Catalogue: which he has not done. But in his Catalogue among Montanus's works he mentions his Apologeticum testimonium, at the [en]d of his Liber generationis et regenerationis Adami: but 'tis an Error either of his own or the printers'. The Tract is a Poem, entitled Apostolicum Testimonium (not Apologeticum) in 2 parts. 'tis an account of the first 7 Chapters of the Acts of the Apostles in verse. ${ }^{22}$
\end{abstract}

Así pues el error del que, según Mill, podría venir la confusión y la búsqueda sin resultados de su destinatario es que James, autor también del primer catálogo impreso de libros de la Bodleian, se equivocó al transcribir el título de una obra impresa de Montano, asignando como título Apologeticum testimonium a un escrito de Montano que en realidad se titulaba Apostolicum testimonium. Mill confiesa no haber leído el pasaje de la otra

19 El escrito se conserva en los ficheros de la sala de manuscritos occidentales y de libros impresos antes de 1640, llamada Duke Humfrey's Library, y catalogado en Thonlae Tanneri Index Codicum MSS como "MS. Tanner. 290/fol. 184. Dr. John Mill, to [the secretary to archbp. Sancroft?]".

${ }^{20}$ Ofrecemos nuestra propia edición del pasaje. La carta ha sido también objeto de estudio por parte de M. V. SpotTorno, "Arias Montano en una carta de John Mill”, Sefarad, 50-2, 1990, 475-479. de 1612 .

${ }^{21}$ Es decir, Thomas James en su citada obra A Treatise of the Corruption of Scripture,

22 De MS. Tanner 290, fol. 184r. 
obra de James donde se hacía referencia a la apología de Montano (véase el pasaje procedente del libro titulado A Treatise of the Corruption of Scripture..., citado más arriba). Y, si lo hubiera hecho, habría comprendido que el manuscrito por el que preguntaba su corresponsal era un documento muy diferente a un texto impreso en una de las conocidas obras de Montano.

Un siglo después, el bibliófilo español Gregorio Mayans y Siscar estaba inmerso en sus investigaciones sobre la Biblia Políglota Complutense y la de Amberes. A principios de 1749, en una de sus cartas al noble inglés John Carteret, conde de Granville ${ }^{23}$, Mayans le pedía ayuda para conseguir una copia de la apología montaniana que presuntamente se encontraba en Oxford $^{24}$. Seis años después vuelve a referirse a este documento en una carta a Beniamin Keene, embajador de Gran Bretaña en España, fechada el 25 de mayo de 1754.

Ab accusatione apud Romanum Pontificem facile se Montanus expediuit, scripta Apologia, cuius meminit Paulo Colomesius in Opusculis, cap. 34, ita scribens: "Ob praeclaram illam operam in Bibliorum Regiorum editionem insumtam, Benedictus Arias Montanus haeresium apud Romanum Pontificem postulatus, Apologiam hispanice scripsit, quam in expugnatione Caletana ante annos aliquot inuentam Angli in patriam suam detulerunt, atque in Oxoniensi Bibliotheca tanquam pretiosissimum $\kappa \varepsilon \iota \mu$ í $\lambda$ เov etiam nunc asseruant". ${ }^{25}$

Hasta aquí Mayans se hace eco de la noticia de Colomesio. En las líneas siguientes, el bibliófilo valenciano admite haber pedido ya en varias ocasiones al embajador que le ayudara a localizar este documento en Oxford. Las pesquisas, de nuevo, no tuvieron éxito:

Atque haec est Benedicti Ariae Montani Apologia quam ego aliquando uolui tuo beneficio obtinere, quaeque malo meo et publico non apparuit in Bibliotheca Oxoniensi. Vtinam aliquando incidat in uiri probi manus qui eam sapientiae studiosis non inuideat. ${ }^{26}$

23 Este noble inglés, admirador de la obra cervantina, fue el promotor de la Vida de Miguel de Cervantes Saavedra, escrita por Mayans y publicada en Londres en 1738.

24 Debemos esta noticia a V. Peset Llorca, Gregori Mayans i la cultura de la il.lustració... amb un próleg d'Antoni Mestre, Barcelona, 1975, consultada en http://bivaldi.gva.es/va/corpus/ unidad.cmd ?idUnidad $=56742 \&$ idCorpus $=20000 \&$ posicion $=1$.

25 Traducción: "Montano salió airoso de esta acusación ante el Romano Pontífice tras escribir una apología, de la que se acuerda Paulo Colomesio en sus Opuscula, cap. 34, que dice así: 'A causa de aquella ilustre obra - dice - emprendida para la edición de la Biblia Regia, Benito Arias fue acusado de varias herejías ante el Romano Pontífice y escribió una apología en español, que los ingleses encontraron hace algunos años en el sitio de Calais y llevaron a su patria, y conservan aún en la Biblioteca de Oxford como un preciosísimo tesoro'”.

26 Traducción: "Y esta es la apología de Benito Arias Montano que algunas veces he querido obtener por medio de usted, y que para mi desgracia y la del público no ha aparecido en la Biblioteca de Oxford. Ojalá caiga alguna vez en las manos de un buen señor que no la niegue a los estudiosos del saber". El texto de la carta de Mayans a Keene procede de M. A. SÁNCHEZ Manzano, Prefacios de Benito Arias Montano a la Biblia Regia de Felipe II, León, Universidad de León, 2006, pp. 277-311 (p. 289). Sánchez Manzano traduce "beneficio tuo" como "para usted" en vez de "por medio de usted", interpretación de la que discrepamos, dado que fue el embajador de Inglaterra en España quien, en buena lógica, prestó ayuda Mayans para tratar de localizar el manuscrito en Oxford. 
En las páginas 455-456 del número 56 de la "Gazeta de Sevilla", publicado el viernes 28 de junio de 1811, se lee una Necrología de Arias Montano, cuya primera parte se titula "Noticia histórica de Benito Arias Montano", incluyendo datos biográficos y una información decisiva para comprender las razones del traslado de los restos de Arias Montano a la catedral de Sevilla en $1811^{27}$. Entre la información aportada en esta biografía se halla de nuevo una referencia a la apología, cuya fuente parece ser la ya citada entrada de la Bibliotheca hispana noua de Nicolás Antonio, pues no aporta nada nuevo a la misma:

A pesar de su modestia, tuvo que escribir una apología en su favor, guardado como un riquísimo tesoro en la Universidad de Oxford, adonde lo llevaron los ingleses después de perdida Calés. ${ }^{28}$

También a principios del siglo XIX, hemos hallado el curioso caso de un estudioso tan desesperado por hallar nuestro documento manuscrito que recurrió a un anuncio por palabras en la edición del Morning Chronicle del viernes 17 de noviembre de 1820 , página $3^{29}$ :

D. Nicolas Antonio, in the first volume of the Bibliotheca Noua Hispana, article Benedictus Arias Montanus, after giving an account of the other works of that autor, makes the following remark: "Apologiae pro se scriptae memoriam tantum reliquit, cuius mentionem reperi nuper in opusculis Pauli Colomesii ante paucos annos editis Parisiis 1668, in 12, ob praeclaram illam, ait, operam in Bibliorum Regiorum editionem insumptam Benedictus Arias uariarum haeresium apud Rom. Pontificem postulatus Apologiam Hispanice scripsit, quam in expugnatione Caletana ante aliquot annos inuentam Angli in patriam suam detulerunt atque in Oxoniensi Bibliotheca, tamquam praetiosissimum keimelion etiamnum asseruant. Exoriatur tandem aliquis qui eius editionem procuret!"

The writer of this article, having been unsuccessful in all his attempts to discover the particular library of Oxford, in which the above MS. is preserved, would esteem it a particular favour if any literary Gentleman could commu-

27 J. M. ${ }^{\text {a }}$ Maestre Maestre ha demostrado que la publicación de este texto responde a dos decretos de José Napoleón, uno de 6 de marzo de 1809 y otro de 21 de junio de 1810, en los que se disponía que las cenizas de los varones ilustres que se hallaran en las iglesias reformadas se trasladasen a las catedrales. En realidad, en una muy bien urdida maniobra propagandística, el traslado de los restos mortales de Arias Montano, como el de otros insignes sabios, fue utilizado para maquillar las sacrílegas profanaciones de tumbas que los franceses cometieron en Sevilla al igual que en el resto de las ciudades españolas. Cf. J. M. ${ }^{a}$ MaEstre Maestre, "El traslado de los restos mortales de Benito Arias Montano desde el convento de Santiago de la Espada a la Catedral de Sevilla en 1811", en F. Grau Godina, J. M. ${ }^{a}$ Maestre Maestre, J. Pérez Durà (eds.), Litterae humaniores del Renacimiento a la Ilustración. Homenaje al Profesor José María Estellés, Valencia, Universitat de València, 2009, pp. 363-390.

28 Cf. F. CAlero, J. González manzanares, "Tres aportaciones a la vida y obra de Benito Arias Montano", en J. M. a Maestre Maestre, E. Sánchez Salor, M. A. Díaz Gito, L. Charlo Brea, P. J. Galán Sánchez (eds.), Benito Arias Montano y los humanistas de su tiempo, 2 vols., Mérida, Editora Regional de Extremadura / Instituto de Estudios Humanísticos, 2006, vol. I, pp. 57-64 (p. 59).

${ }^{29} \mathrm{El}$ documento se puede encontrar digitalizado en el siguiente sitio web: http://news paperarchive.com/uk/middlesex/london/morning-chronicle/1820/11-17/page-3. 
nicate to the Editor of this Journal (The Morning Chronicle) any information respecting it.

Ya en el siglo XX, Marcelino Menéndez Pelayo apunta de nuevo en su Biblioteca de traductores españoles 30: "Dícese que en la Biblioteca de Oxford se guarda una Apología de la Biblia Regia hecha por A. Montano". La localización del preciado documento ha ocupado también a expertos conocedores de la Bodleian como J. N. L. Myres, quien en su artículo titulado "Thomas James and the painted friese" 31 , hace referencia a la admiración que profesaba hacia Arias Montano el primer bibliotecario de la Bodleian, concluyendo que la apología "cannot now be traced in Bodley".

\section{Conclusiones}

La apología en español escrita por Arias Montano en defensa de la lengua hebrea, de la Biblia Políglota y de sí mismo tuvo una azarosa y desafortunada transmisión: la única copia de la que nos han llegado noticias fue robada por los ingleses en el saqueo de Cádiz (1596) y depositada a finales del siglo XVI por George Ryves, alcalde del New College, en alguna Biblioteca de la Universidad de Oxford. Al menos hasta 1612 Thomas James, primer bibliotecario de Oxford, pudo acceder al documento, del que ofrece una vaga información sobre su contenido. Sin embargo, en un momento determinado y por razones que desconocemos, este manuscrito parece haber desaparecido de los anaqueles de la Bodleian. En una carta de Edward Browne al Dr. Gale, fechada el 4 de junio de 1697, criticando la falta de seguridad en la conservación de valiosos manuscritos conservados en Inglaterra, ya da por perdida la apología de Montano:

And the University of Oxford presently lost the Acts of the Inquisition against Arias Montanus and his apology for himself, when once Dr. James gave notice of it in his Corruption of the Fathers. ${ }^{32}$

A pesar de todo, la importancia de este documento ha llevado a un buen número de investigadores a seguir su rastro de forma impenitente desde los tiempos de Nicolás Antonio hasta el día de hoy. Los métodos de prospección han sido extremadamente variados. Al fin y al cabo, cuando se han agotado ya las principales herramientas que ayudan a rastrear documentos manuscritos, todas las demás medidas son también válidas: notas de prensa como las del Morning Chronicle (hoy contamos con blogs y sitios web), cartas a embajadores, archivistas y bibliotecarios y, por su puesto, trabajo en red.

30 Santander, CSIC, 1951-1952, vol. I, p. 163.

31 The Bodleian Library Record, 4-1, Abril 1952, 30-51 (p. 45 y nota 1).

32 Memoirs of Samuel Pepys, Secretary to the Admiralty in the Reigns of Charles II and James II, Comprising His Diary from 1659 to 1669, Deciphered by the Rev. John Smith and a Selection from his Private Correspondence, Edited by Richard Lord Braybrooke, 5 vols., Londres, 1828 , vol. V, p. 237. 
Para terminar, es necesario plantear aquí de forma explícita la pregunta que el avezado lector seguramente tenga en mente desde varias páginas atrás: ¿no deberíamos aceptar ya el hecho de que la apología de Arias Montano se ha perdido para siempre, como ha ocurrido con otros muchos valiosos documentos de principios de la Edad Moderna?

Por ahora, lo único que puede decir quien escribe estas líneas es que sigue buscando este manuscrito en las bibliotecas de la Universidad de Oxford cada vez que tiene ocasión.

En los catálogos de manuscritos de la Bodleian, el único regalo identificable de George Ryves a la Bodleian es una biblia del siglo XII en dos volúmenes (MSS Auct. E. infra 1-2) ${ }^{33}$ : pero se trata de una biblia latina de origen inglés que Ryves había obtenido de la Catedral de Winchester - nada que ver, por lo tanto, con España. También he realizado prospecciones, con la ayuda del personal de biblioteca, entre los fondos de New College, de donde fue alcalde Ryves, quien no dejó ningún manuscrito a ese College; tampoco en los catálogos correspondientes ${ }^{34}$ hay ninguna referencia a un manuscrito español relacionado con Montano. He recurrido, por último, al personal del Department of Special Collections and Western Manuscripts, incluyendo el director de catalogación y conservación de manuscritos de la Temprana Edad Moderna, que han hecho todo lo posible por orientarme entre las colecciones de esa magnífica biblioteca.

Los esfuerzos, por el momento, han sido en vano. Pero la esperanza es lo último que se pierde, sobre todo tratándose de un texto de tal transcendencia que, sin haber sido nunca localizado ni publicado, ha despertado tanto interés entre los principales estudiosos del Humanismo español. Por todo lo anterior, debo cerrar el presente trabajo expresando yo también que consideraría un favor particular si algún colega, bibliotecario o coleccionista privado pudiera comunicarme alguna nueva información sobre este precioso documento.

ABSTRACT: Scholars dealing with Humanism can access the most important Early Modern handwritten documents by using various tools and finding aids: library catalogues, catalogues of manuscripts and printed books, bio-bibliographical indexes and also the growing secondary literature (books and articles) on that subject. Yet one often comes across information about valuable manuscripts whose whereabouts is not known to us anymore. In this article I present the case of an important vindication of the Royal Bible written by the Spanish scholar Benito Arias Montano: the only copy of this document known to us was sacked by the English and taken to the library of Oxford, where it has not been found until today by a long chain of scholars who have tried to locate it by all means since the seventeenth century.

KEY wORDS: Humanism; Biblical Scholarship; Arias Montano.

33 Véase descripción bibliográfica en el catálogo de manuscritos de la Bodleian titulado A Summary Catalogue of Western Manuscripts in the Bodleian Library at Oxford Which Have Not Hitherto Been Catalogued in the Quarto Series: With References to the Oriental and Other Manuscripts, 7 vols. en 8, Oxford, Clarendon Press, 1895-1953, vol. II: 1, p. 358, entrada número 2426-7.

${ }^{34}$ Cf. Henry O. Coxe, Catalogus codicum MSS. qui in collegiis aulisque Oxoniensibus hodie adseruantur, 2 vols., Oxford, 1852. El volumen I incluye los manuscritos del New College. 


\section{I \\ COMMENTATIONES}

Antígona: luzes e sombras - ANA PaUla Pinto

Qui sont les rotae dans les Res Rusticae (II, 1, 5) de Varron? - Marcel Meulder ........ 31

Athènes historique, Athènes éternelle. Le regard de Plutarque sur la ville et ses monuments - FrançOISE FraZIER

La mirada de Plutarco: significados y funciones de su testimonio visual en las Vidas Paralelas - CARLOS ALCALDE-MARTín

Tra Costantinopoli e Vivarium: fonti greche e fonti latine nel Commento ai Salmi di Cassiodoro - PATRIZIA StOPPACCI.

Los progymnasmata de Aftonio publicados por Palmireno en 1552: estudio de un ejemplar localizado en la Biblioteca Nacional de Portugal - M.A Violeta Pérez CUstodio

En la estela de Horacio: una epístola latina inédita de Giacinto Frangipane

- EduARdo del Pino González

Los progymnasmata en los Praeceptionum rhetoricarum libri $V$ et exercitationum libri II de Georg Henisch: fuentes y materiales - GREGORIO RODRíGUEz HERRERA

Entre a fidelidade às origens e o contexto interpretativo: duas adaptações portuguesas contemporâneas da tragicidade ambígua de Antígona - MARIA José FERREIRA LOPES .

\section{II \\ STVDIA BREVIORA}

El análisis de los datos sobre la religión prehelénica: una cuestión metodológica Marcello Tozza.....

Sobre la prudencia de un Cicerón demasiado hábil: De inventione 1, 49, 91 a propósito de Cornelia, madre de los Gracos - MARc MaYer y Olivé.

Virgil's Smooth-Talking Pygmalion and Jerome's Commentaries on Mordiloquent Minor Prophets - NeIL AdKIN......

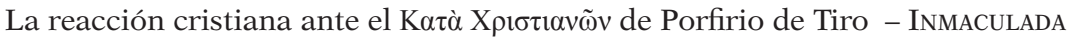
Rodríguez MORENO.

El viaje de Trezenzonio a la isla de Solistición. Refacción de material y distintos niveles de sentido - Joel VARELA RodRíGUEZ 
La Austriaca siue naumachia de Francisco de Pedrosa y la propaganda al servicio del poder - JuAN CARLos Jiménez del CASTILlo .....

La apología de la Biblia Regia escrita por Benito Arias Montano: un documento en paradero desconocido - ANTONIo Dávila Pérez

El descenso al infierno del Ovidio de Vintila Horia en la novela Dios ha nacido en el Exilio. Diario de Ovidio en Tomis - AlEJANDro Martínez Sobrino.

\section{VARIA NOSCENDA}

Iscrizioni romane di tradizione manoscritta: il codice epigrafico di Aquiles Estaço - Alejandra Guzmán Almagro ....

\section{IV}

\section{LIBRI RECENSITI}

\section{a) Edições de texto. Comentários. Traduções. Estudos Linguísticos}

Esouilo, Tragedias IV: Coéforos. Euménides, introducciones y texto por Francisco Rodríguez Adrados, traducciones y notas por Esteban Calderón Dorda - RUI Miguel DuARTe

Sófocles, Icneutas, os sátiros rastreadores: fragmentos de um drama satírico reconstituído para a contemporaneidade com base nos aparatos de Stefan Radt e Hugh Lloyd-Jones. Tereza Virgínia Ribeiro Barbosa (org.) - Sofia Frade ....

María Teresa Gallego Pérez, Vida y muerte en el Corpus Hippocraticum - Maria José Mendes e Sousa

PolyBius, The Histories, vol. VI: books 28-39, translated by W. R. Paton, revised by Frank W. Walbank and Christian Habicht; Unattributed fragments, edited and translated by S. Douglas Olson - Rui Miguel DuARTE

Marco Terêncio Varrão, Das coisas do campo. Tradução, introdução e notas de Matheus Trevizam - Manuel José De Sousa BARBosa

VIRGílio, Geórgicas I. Org. Matheus Trevizam, traduções de António Feliciano de Castilho e Matheus Trevizam - ANA FILIPA Gomes FerReIRA

Tibulo, Poemas (Cantos de Amores). Tradução, introdução e notas de Carlos Ascenso André - Luís CERqueira.

Francesco Citti, Cura sui. Studi sul lessico filosofico di Seneca - Ana Filipa Silva.......

Sandrine Dubel (ed.), Jackie Pigeaud (postfac.), Lucien de Samosate. Portrait $d u$ Sophiste en Amateur d'Art - Nuno SimõEs Rodrigues 
Juan Antonio López Férez (ed.), Galeno. Lengua, composición literaria, léxico, estilo - InMACUlada Rodríguez Moreno .....

S. Douglas Olson (ed.), Athenaeus: The Learned Banqueters. Volume VII: Books 13, 594b-14 - Fotini HaDJITTOFI

CALCIDio, Traducción y Comentario del Timeo de Platón. Introducción, traducción y notas de Cristóbal Macías Villalobos - INÊs BoLINHAS

Nonnus of Panopolis, Paraphrasis of the Gospel of John XI. Edited by Konstantinos Spanoudakis - Fotini HadjITTOFI.

Enara San Juan Manso, El Commentum Monacense a Terencio - Eftychia BATHRELLOU .

Navigatio sancti Brendani, Alla scoperta dei segreti meraviglosi del mondo, edizione critica a cura di Giovanni Orlandi e Rossana E. Guglielmetti, introduzione di Rossana E. Guglielmetti, traduzione italiana e commento di Giovanni Orlandi - ARNALDo Do EsPíRITO SANTO.

D. Jerónimo Osório, Opera Omnia. Tomo II: Epistolografia. Estabelecimento do texto latino por Sebastião Pinho e António Guimarães Pinto. Introdução, tradução, notas e comentários de António Guimarães Pinto - MARIa Luísa RESENDE.

D. Jerónimo Osório, Opera Omnia. Tomo III: Comentários aos Provérbios de Salomão. Estabelecimento do texto latino por Sebastião Pinho e António Guimarães Pinto. Introdução, tradução, notas e comentários de António Guimarães Pinto - Maria Luísa Resende....

\section{b) Literatura. Cultura. História}

António José Gonçalves de Freitas, Os Deuses e a Origem do Mundo - Maria João CORREIA SANTOS.....

Franco Montanari, Stephanos Matthaios, Antonios Rengakos, Brill's Companion to Ancient Greek Scholarship, vol. 1: History. Disciplinary profiles; vol. 2: Between theory and practice - Nereida Villagra .....

Richard Bouchon, P. Brillet-Dubois, Nadine Le Meur-Weissman (eds.), Hymnes de la Grèce Antique. Approches littéraires et historiques. Actes du Colloque international de Lyon, 19-21 juin 2008 - ABEL N. PENA

André Laks, Rossella Saetta Cottone (dir.), Comédie et philosophie. Socrates et les 'Présocratiques' dans les Nuées d'Aristophane - Rui Miguel DuARTE

Victoria Wohl, Euripides and the Politics of Form - SofIA Frade

Juan Antonio López Férez, Mitos en las obras conservadas de Eurípides. Guía para la lectura del trágico - José Vela TeJADA 
Robert Mayhew, The Aristotelian Problemata Physica. Philosophical and Scientific Investigations - BERNARDO MACHADO MOTA

André Hurst, Dans les marges de Ménandre - EfTychia Bathrellou

Joaquim S. Pinheiro, Tempo e espaço da paideia nas Vidas de Plutarco - Ramiro GonZÁlez Delgado

Joseph GeIger, Hellenism in the East. Studies on Greek Intellectuals in Palestine FOTINI HADJITTOFI

Gesine Manuwald, Astrid Voigt (eds.), Flavian Epic Interactions - Ana Maria Lóio...

Yvan Nadeau, Dog Bites Caesar! A Reading of Juvenal's Satire 5 (with Horace's Satires I, 5; II, 5; II, 6; Epistles I, 1; I, 16; I, 17) - Maria CRIstina Pimentel

Andrea LaI, Alle nozze dello Sposo. Gregorio Magno commentatore del 'Cantico dei cantici' e le sue fonti - AMÉRICO PEREIRA

Cécile Bost-Pouderon, Bernard Pouderon (eds), Les Hommes et les Dieux dans l'ancien roman. Actes du colloque de Tours, 22-24 octobre 2009 - GIUSEPPE CiafARDONE

Cristina-Georgeta AleXandrescu (ed.), Cult and votive monuments in the Roman Provinces, Proceedings of the $13^{\text {th }}$ International Colloquium on Roman Provincial Art (Bucharest, Alba Iulia, Constanta, 27 $7^{\text {th }}$ May $-3^{\text {rd }}$ June 2013 - within the framework of Corpus Signorum Imperii Romani) - MARIA João CorreIA SANTOS

Rosario Moreno Soldevila, Juan Martos (eds.), Amor y sexo en la literatura latina - Maria JoÃo Correia Santos

Juan Antonio López Férez (ed.), La comedia griega en sus textos. Forma (lengua, léxico, estilo, métrica, crítica textual, pragmática) y contenido (crítica política y literaria, utopía, sátira, intertextualidad, evolución del género cómico) - José VELA TEJADA

JoËL Thomas, Mythanalyse de la Rome Antique. Pref. Paul Veyne - Nuno Simões RODRIGUES

Aline Estèves, Jean Meyers (eds.), Tradition et innovation dans l'épopée latine, de l'Antiquité au Moyen Âge - Luís M. G. CERoueIra....

Arnaud Perrot (ed.), Les Chrétiens et l'Hellénisme. Identités religieuses et culture grecque dans l'Antiquité tardive - RuI Miguel DuARTE

STÉPHANE RATTI, Antiquus error: Les ultimes feux de la résistance païenne 'Scripta uaria' augmentés de cinq études inédites - MARIA JoÃo CoRrEIA SANTOS.

António Manuel lopes Andrade, Carlos de Miguel Mora, João Manuel Nunes TORRÃo (coords.), Humanismo e ciência. Antiguidade e Renascimento - LuANA GIURGEVICH 
Belmiro Fernandes Pereira, Retórica e Eloquência em Portugal na Época do Renascimento - MARIA Luísa ReSENDE..

Alejandro Coroleu, Printing and Reading Italian Latin Humanism in Renaissance Europe (ca. 1470 - ca.1540) - MARIA Luísa RESENDE

A. Castro Santamaría, J. Garcia Nistal (coords.), La impronta humanística (ss. XV-XVIII), saberes, visiones e interpretaciones - MADALENA BRITO.

Jerry Toner, Homer's Turk: How Classics Shaped Ideas of the East - RuI Carlos FONSECA

Sergio Audano, Classici lettori di classici. Da Virgilio a Marguerite YourcenarGiUSEPPE CiAfARDONE

Timothy Saunders, Charles Martindale, Ralph Pite, Mathilde Skoie (ed.), Romans and Romantics - RICARDO NOBRE

Francisco Rodríguez Adrados, El cuento erótico griego, latino e índio. Ilustrações de Antonio Mingote - Hiteshrumar Parmar

Francisco Rodríguez Adrados, El Río de la Literatura. De Sumeria y Homero a Shakespeare y Cervantes - JoAQuim PINHEIRO.

Belmiro Fernandes Pereira, Jorge Deserto (ed.), Symbolon III: Paz e Concórdia; Belmiro Fernandes Pereira, Ana Ferreira (ed.), Symbolon IV: Medo e Esperança - RicARDO NOBRE.

Cristina Santos Pinheiro, Anne Martina Emonts, Maria da Glória Franco, Maria JoÃo Beja (coords.), Mulheres: Feminino, Plural - Vanda Anastácio.....

Il senso del Medioevo: In memoriam di Claudio Leonardi, a cura di Antonella Degl'Innocenti, Donatella Frioli, Paolo Gatti, Fabrizio Rasera - ManUEL José DE Sousa Barbosa

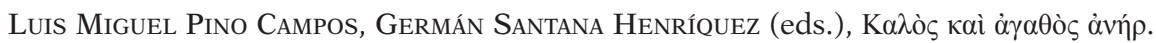

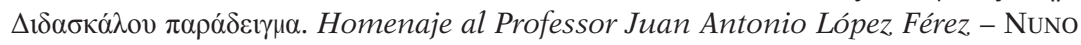
SIMÕES RODRIGUES.

IÑIgo Ruiz ARZalluz (coord.), Estudios de Filología e Historia en Honor del Profesor Vitalino Valcárcel, 2 vols., edição de Alejandro Martínez Sobrino, Maria Teresa Muñoz García de Iturrospe, Iñaki Ortigosa Egiraun e Enara San Juan Manso Maria Fernandes.

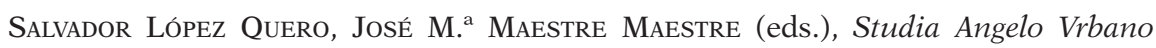
Dicata - ANDRÉ SimõES .....

Cristóbal Macías Villalobos, José M. ${ }^{a}$ Maestre Maestre, Juan F. Martos Montiel (eds.), Europa Renascens. La Cultura Clásica en Andalucía y su proyección europea - ANDRÉ SIMÕES. 
Este XLIV volume da Nova Série de Euphrosyne foi composto, impressso e encadernado em Braga, nas Oficinas da PUBLITO - Estúdio de Artes Gráficas, Lda.

Dezembro de 2016 


\title{
E V P H R O S Y N E
}

\author{
REVISTA DE FILOLOGIA CLÁSSICA \\ Centro de Estudos Clássicos - Faculdade de Letras \\ PT - 1600-214 LISBOA \\ centro.classicos@letras.ulisboa.pt
}

\section{ARTICLE SUBMISSION GUIDELINES}

1. Euphrosyne - Revista de Filologia Clássica, the peer journal of the Centre for Classical Studies, publishes papers on classical philology and its disciplines (including classical reception and tradition).

2. Papers can be sent to centro.classicos@letras.ulisboa.pt or to the Centre for Classical Studies' post mail.

3. Papers submitted: must be original; cannot be yield to other entity; must be sent in their definite version; have to be presented according to these guidelines; will not be returned to the author. Papers will be submitted to peer reviews.

4. Papers will be accepted until $31^{\text {st }}$ of December in the year previous to publication; an acceptance notification will be sent to the author until $30^{\text {th }}$ of April in the year of publication.

5. Originals must always be submitted in double electronic format (Word/.doc(x) and PDF).

6. Papers must have: a) title (short and clear); b) author's name and surname; c) author's academic or scientific institution; d) author's email; e) abstract (10 lines) in English; f) three key-words in English.

7. Recommended size is 10 pages and never more than $20 \mathrm{~A} 4$ pages (font size 12 , double spaced).

8. Notes: endnotes, with sequential numeration. When published, these will be converted to footnotes.

9. References:

a) Remissions to pages within the paper are not allowed.

b) Note references:

Books: J. DE RomilLy, La crainte et l'angoisse dans le théâtre d'Eschyle, Paris, Les Belles Letres, 1959, pp. 120-130; $2^{\text {nd }}$ reference: J. DE RomiLlY, op. cit., p. 78.

Journals: R. S. CALDwelL, "The Misogyny of Eteocles", Arethusa, 6, 1973, 193-231 (vol., year, pp.). $2^{\text {nd }}$ reference: R. S. CALDWELL, loc. cit.

Multi-author volumes: G. Cavallo, "La circolazione dei testi greci nell'Europa dell'Alto Medioevo" in J. Hamesse (ed.), Rencontres de cultures dans la Philosophie Médiévale - Traductions et traducteurs de l'Antiquité tardive au XIVe siècle, Paris, Les Belles Letres, 1971, pp. 47-64.

c) Abbreviations: to Latin authors will be followed ThLL conventions; Liddel-Scott-Jones will be used to Greek authors; Année Philologique to abbreviate journal titles; common abbreviations: p./pp.; ed./edd.; cf.; s.u.; supra; op. cit.; loc. cit.; uid.; a.C./d.C. (roman).

d) Quotations: Must be marked by quotes “..." (but not in Greek); italic is used to highlight words or short sentences; quotations in Latin or Greek must be brief.

10. Images must have quality (preferably in TIF format, minimum resolution 200 p.p.), provided in electronic format, with the precise indication of where they must be placed in the text, and who is their author. The author is responsible for obtaining any copyrights needed.

11. The author will not be provided with more than one set for review, which has to be returned within a week period. Originals cannot be modified.

12. Authors will receive a physical copy of the volume and the electronic version of their paper. 